\title{
SOIL SALINITY MAPPING USING MULTITEMPORAL LANDSAT DATA
}

\author{
A. Azabdaftari ${ }^{\text {a }}$, F. Sunar ${ }^{\text {b }}$ \\ ${ }^{a}$ Istanbul Technical University, Informatics Inistitue, Communication Systems Dept.,34469, Maslak Istanbul, Turkey \\ azabdaftari@itu.edu.tr \\ ${ }^{\mathrm{b}}$ Istanbul Technical University, Civil Engineering Faculty, Geomatics Engineering Dept., 34469, Maslak Istanbul, Turkey \\ fsunar@itu.edu.tr
}

Commission VII, WG VII/1

KEY WORDS: Remote sensing, Soil salinity, Landsat data, Salinity index, Vegetation index

\begin{abstract}
:
Soil salinity is one of the most important problems affecting many areas of the world. Saline soils present in agricultural areas reduce the annual yields of most crops. This research deals with the soil salinity mapping of Seyhan plate of Adana district in Turkey from the years 2009 to 2010, using remote sensing technology. In the analysis, multitemporal data acquired from LANDSAT 7$\mathrm{ETM}^{+}$satellite in four different dates (19 April 2009, 12 October 2009, 21 March 2010, 31 October 2010) are used. As a first step, preprocessing of Landsat images is applied. Several salinity indices such as NDSI (Normalized Difference Salinity Index), BI (Brightness Index) and SI (Salinity Index) are used besides some vegetation indices such as NDVI (Normalized Difference Vegetation Index), RVI (Ratio Vegetation Index), SAVI (Soil Adjusted Vegetation Index) and EVI (Enhamced Vegetation Index) for the soil salinity mapping of the study area. The field's electrical conductivity (EC) measurements done in 2009 and 2010 , are used as a ground truth data for the correlation analysis with the original band values and different index image bands values. In the correlation analysis, two regression models, the simple linear regression (SLR) and multiple linear regression (MLR) are considered. According to the highest correlation obtained, the 21st March, 2010 dataset is chosen for production of the soil salinity map in the area. Finally, the efficiency of the remote sensing technology in the soil salinity mapping is outlined.
\end{abstract}

\section{INTRODUCTION}

Soil salinity is one of the widespread environmental hazards all around the world, especially in arid and semiarid regions. Soil salinization that mainly occurs due to irrigation and other intensified agricultural activities, is one of the most severe problems among the many forms of soil degradation (Akramkhanov, 2011). The development of saline soils is a dynamic phenomenon, which needs to be monitored regularly in order to secure up to date knowledge of their extent, degree of severity, spatial distribution, nature and magnitude. For monitoring dynamic processes, like salinization, remotely sensed data has great potential; it uses aerial photography, thermal infrared or multispectral data acquired from platforms such as Landsat satellite (Allbed and Kumar, 2013). Previously, soil salinity has been measured by collecting soil samples in the region of interest, and then the samples were analyzed in laboratory in order to determine the amount of electric conductivity in the soil but this method was time and cost consuming. However, remote sensing data offer more efficiently and economically rapid means and techniques for monitoring and mapping soil salinity. There are many satellites and sensors, which are useful in detecting and monitoring the saline soil. Multispectral data such as LANDSAT, SPOT, IKONOS, EO1, IRS, and Terra-ASTER with the resolution can be ranged from medium to high as well as hyperspectral sensors.
The sensors scan only the soil surface, while the entire soil profile is involved and should be considered. This limitation highlights the necessity of using other data and techniques, in combination with remote sensing (Farifteh, 2006). The main objectives of this study are: (i) to understand the spectral reflectance characteristics of saline soil in Seyhan plate, (ii) to explore the potential of Landsat imagery to detect and map the soil salinity over the study area, (iii) to analyse the correlation between field measurements and Landsat imagery, and (iv) to produce the soil salinity map according to high, moderate and low saline content.

\section{STUDY AREA}

Çukurova is a district in south central of Turkey covering the provinces of Adana and Mersin (Figure 1). It is located in the coordinates of $37^{\circ} 02^{\prime} 52^{\prime \prime}$ North latitudes and $35^{\circ} 17^{\prime} 54^{\prime \prime}$ East longitudes. The total area of the Çukurova is about $38,000 \mathrm{~km}^{2}$, Turkey's biggest delta plain with a large stretch of flat and fertile land, which is among the most agriculturally productive areas of the world. The climate is relatively mild and humid in the winter months and the alluvial soils make the area highly suitable for agriculture. Akarsu irrigation basin and Seyhan plate are located in Çukurova plain. 


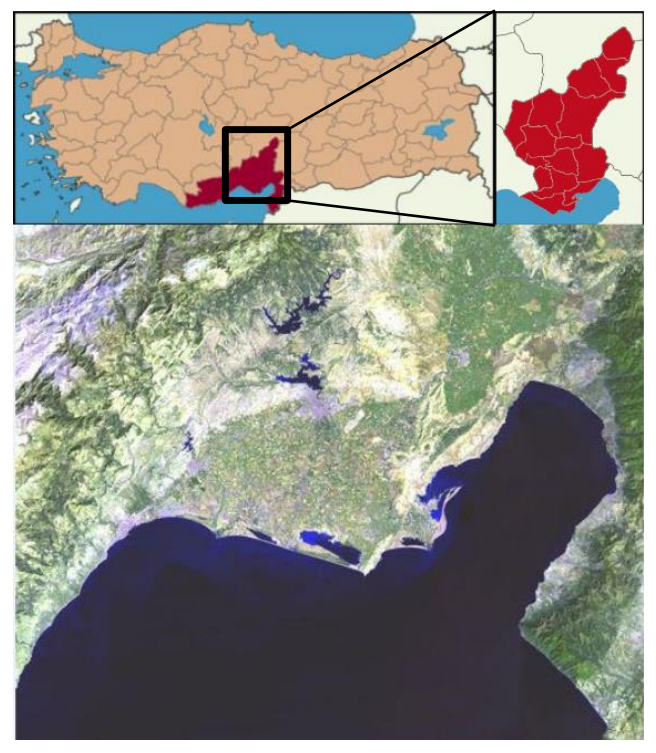

Figure1. The map and satellite image of the study area.

\section{DATA}

\subsection{Satellite data}

In this study, the Landsat $7 \mathrm{ETM}^{+}$satellite images with 30- meter resolution were used. The images were georectified to a Universal Transverse Mercator (UTM) coordinate system, using World Geodetic System (WGS) 1984 datum, assigned to north UTM zone 36 and Path 175 Row 34, 35. The most compatible and close dates were selected according to the dates of field work not to have any problems like seasonal changes. The dates of satellite images used are given in the Table 1.

\begin{tabular}{|c|c|}
\hline $\begin{array}{c}\text { Date of field } \\
\text { measurements }\end{array}$ & $\begin{array}{c}\text { Date of satellite } \\
\text { pass }\end{array}$ \\
\hline $02-05-2009$ & $19-04-2009$ \\
\hline $04-10-2009$ & $12-10-2009$ \\
\hline $04-10-2010$ & $31-10-2010$ \\
\hline $24-03-2010$ & $21-03-2010$ \\
\hline
\end{tabular}

Table 1. The dates of field measurements and Landsat 7 acquisitions.

\subsection{Ground truth measurements}

Fieldwork performed by Cukurova University, Remote Sensing and GIS group in May 2009, October 2009, October 2010 and March 2010, were used in the analysis. In these dates, different numbers of soil samples and soil electrical conductivity (EC) were collected by using the EM-38 device. As a total, 688, 269,153, 27 samples were collected in the dates of 12-Oct-2009, 31-Oct-2010, 19Apr-2009 and 21-Mar-2010, respectively. Dataset consists of four Landsat images belonging to years 2009 and 2010 winter and summer cropping seasons; hence it is possible to evaluate the soil salinity conditions in both seasonal and annual periods.

\section{METHOD}

\subsection{Preprocessing}

As a first step, the preprocessing techniques such as atmospheric and radiometric corrections should be applied to multitemporal Landsat images. Regarding to sensor $\left(\mathrm{ETM}^{+}\right)$, the unstripping of Landsat 7 satellite data was performed as a radiometric correction.

Then, atmospheric correction was applied in order to remove or reduce the influence of the atmosphere. Especially, this correction is recommended to be done in the studies, when the establishment of a relationship between field measurements and spectral reflectance values are needed (Selch, 2012). Reflectance calibration is applied by deriving the reflectance value from the DN and calculating the top of atmosphere reflectance (TOA).

\subsection{Soil salinity indices}

Sixteen various spectral salinity indices developed in numerous studies related to salt detection and soil salinity mapping were examined for all the Landsat images and the most used seven salinity indices (NDSI, SI 1, SI 2,SI 4, SI 9 , SI 10, SI 14) taken into consideration in this study are given in Table 2 .

\begin{tabular}{|c|c|c|}
\hline Salinity indices & Equation & Reference \\
\hline $\begin{array}{c}\text { Normalized } \\
\text { Differential } \\
\text { Salinity Index }\end{array}$ & $N D S I=\frac{(R-N I R)}{(R+N I R)}$ & $\begin{array}{c}\text { (Khan, } \\
\text { Rastoskuev } \\
\text { et al. 2001) }\end{array}$ \\
\hline Salinity Index 1 & $S I=\sqrt{B \times R}$ & $\begin{array}{c}\text { (Khan, } \\
\text { Rastoskuev } \\
\text { et al. 2001) }\end{array}$ \\
\hline Salinity Index 2 & $S I=\sqrt{G \times R}$ & $\begin{array}{c}\text { (Douaoui, } \\
\text { 2006) }\end{array}$ \\
\hline Salinity Index 4 & $S I=\sqrt{G^{2}+R^{2}}$ & $\begin{array}{c}\text { (Douaoui, } \\
\text { 2006) }\end{array}$ \\
\hline Salinity Index 9 & $S I=\frac{\left(B_{5} \times B_{6}-B_{6} \times B_{6}\right)}{B_{5}}$ & $\begin{array}{c}\text { (Bannari, } \\
\text { Guedon et } \\
\text { al. 2008) }\end{array}$ \\
\hline Salinity Index 10 & $S I=\frac{R \times N I R}{G}$ & $\begin{array}{c}\text { (Abbas, } \\
\text { 2007) }\end{array}$ \\
\hline 2007)
\end{tabular}

Table 2: The most common salinity indices used in this study.

\subsection{Vegetation indices}

Vegetation indices can also be used in soil salinity analyses besides the salinity indices since halophytic plants grow 
naturally in saline soils, and can be adapted to high soil salinity. Therefore, vegetation has been used as an indirect indicator to predict and map soil salinity. Four different vegetation indices were applied and among them two most common vegetation indices (Table 3) showing better correlation were taken into consideration.

\begin{tabular}{|c|c|}
\hline $\begin{array}{c}\text { Vegetation } \\
\text { Indices }\end{array}$ & Equation \\
\hline $\begin{array}{c}\text { Normalized } \\
\text { Differential } \\
\text { Vegetation Index }\end{array}$ & $N D V I=\frac{(N I R-R)}{(N I R+R)}$ \\
\hline $\begin{array}{c}\text { Soil Adjusted } \\
\text { Vegetation Index }\end{array}$ & $S A V I=\frac{\mathrm{NIR}-\mathrm{VIS}}{\mathrm{NIR}+\mathrm{VIS}+\mathrm{L} \times(1+\mathrm{L})}$ \\
\hline
\end{tabular}

Table 3. The vegetation indices used in this study.

\subsection{Correlation}

In this step, the correlation between electrical conductivity of the collected field samples and band values of satellite images is carried out to find the relationship between these variables and assess their efficiency in predicting soil salinity using Simple Linear Regression (SLR) and Multiple Linear Regression (MLR) techniques. Regression modeling techniques are widely used for predicting a variable's spatial distribution (Palialexis, 2011).

\subsubsection{Simple linear regression}

The SLR model was applied by using a single band values for predicting soil salinity. To compute the single band correlation, the digital values corresponding to each sample was extracted for each band in the Landsat images and this has been carried out for all data set $(2009$ - 2010) to develop the relationship with in-situ electrical conductivity measurements done in both vertical and horizontal orientations. The same process was applied to the radiometrically corrected TOA value of sample points in order to evaluate the correlation with salinity measurements. The correlation of in-situ EC values with both DN and TOA values was assessed.

The univariate analysis showed that all bands are not statistically significant predictors of salinity, and not any specific differences were found between the correlation of EC value with either DN or TOA in all satellite image dataset. As a second step, twenty indices including sixteen salinity indices and four vegetation indices were used as a part of the correlation analysis. These indices were chosen with regard to the relevant literature based on their efficiencies in the soil salinity mapping. Hence, the correlation analyses between each indices with EC value in each date was performed using linear regression method to investigate the strongest correlation with the sampled soil salinity values. Similar to correlation of DN and EC values, the correlation of different indices with the in-situ soil salinity measurements was not sufficient

While considering the salinity indices, some of them like SI 1, SI 9, and SI 14 on 21st March, 2010 demonstrate higher correlation among all other salinity indices.
The output images of applied indices are given in Figure 2.
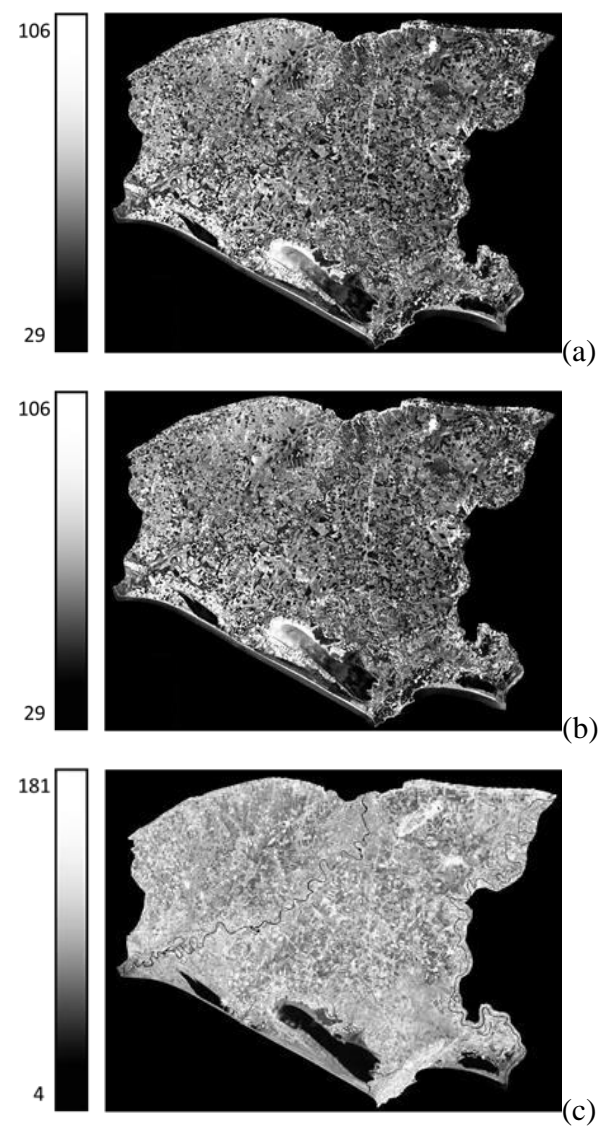

Figure 2. Salinity indices images of Seyhan plate on 21st March, 2010. (a) SI 1 (b) SI 9 (c) SI 14.

\subsubsection{Multiple linear regression}

Since the result of simple linear regression survey was not satisfactory, as a second approach, the multiple linear regression (MLR) technique was applied. In this regression method, pixel values of all six bands of Landsat 7 (except band 6) were used as an independent variable, and EC value of soil was used as a dependent variable.

In multiple regression, two quantitative criteria between measured and predicted values are calculated. $\mathrm{R}^{2}$ values indicate the strength of the statistical linear relationship between measured and predicted soil salinity values and Pvalue tests the hypothesis for each term in linear regression. A low p-value $(<0.05)$ indicates that it is possible to reject the null hypothesis and there is sufficient evidence to conclude that the coefficient does not equal zero and that changes in the predictor are associated with changes in the response variable. In the Minitab software used, high pvalues $(>0.05)$ were regarded as disqualified points and removed from analysis. After removing some sample points multiple regressions was reapplied, until there was not any disqualified sampled point.

Regarding to this step, it is not always very easy to decide which independent variable should be included or removed 
from the regression, since the use of too much variables may lead to poor prediction. Therefore, stepwise regression was used as a solution to overcome this issue.

Among all the correlation results, in both multiple regression and stepwise method, the correlation of $21 \mathrm{st}$ March, 2010 is the highest. The correlation coefficient of six bands of Landsat images dated 21st March, 2010 with the EC value in vertical orientation was $36.56 \%$; however after removing some of the sampled points, the $\mathrm{R}^{2}$ value has increased to $78.40 \%$. Normal probability plot is shown in Figure 3.

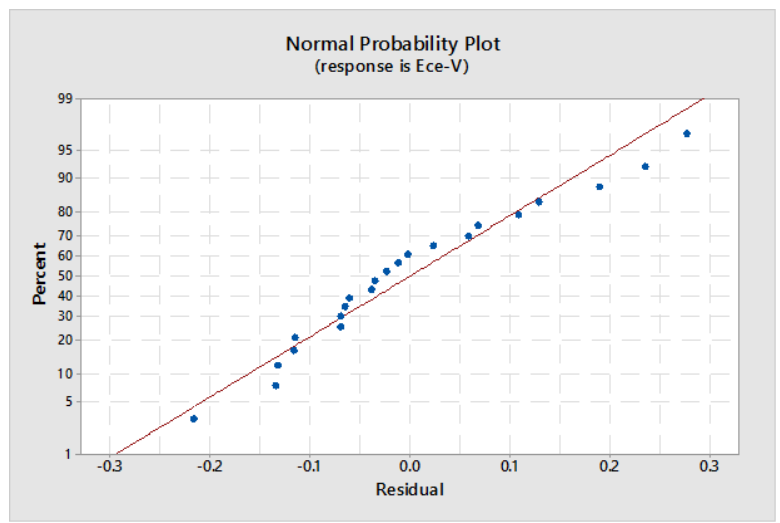

Figure 3. Multiple linear regression result of 21st March, 2010 with all bands and 22 sampled points.

Regression equation obtained is given below:

Predicted Soil Salinity $=2.24-0.0341$ B1 -0.0093 B2

+0.0576 B3 + 0.00125 B4 - 0.0089 B5 - 0.0410 B6

\subsubsection{Multiple linear regression with highest correlated bands and indices}

As a final approach, multiple regression analysis was conducted using different combinations of 1) individual bands having the highest correlation, 2) salinity indices 3) vegetation indices with $\mathrm{EC}$ value. The purpose of this analysis is to select only the bands that have high correlation with the in-situ data, i.e. the following set of bands given in Table 4 were selected for each image and these band combinations were used as independent variables (Azabdaftari, 2015)

\begin{tabular}{|c|lr|}
\hline Satellite date & $\begin{array}{l}\text { Satellite band } \\
\text { Combinations }\end{array}$ & + Salinity index \\
\hline 19-Apr-2009 & Band 4 & SI 4 and SI 9 \\
\hline 12-Oct-2009 & Bands 1 and 2, & SI 1 and SI 2 \\
\hline 21-Mar-2010 & Bands 2, 5 and 6, & SI 4 and SI 9 \\
\hline 31-Oct-2010 & Band 3 & SI 10 \\
\hline
\end{tabular}

Table 4. Different set of band combinations.

For both multiple regression and stepwise method, the highest correlation value was observed in the image dated 21st March, 2010. The correlation was calculated among
Band 2, Band 5 and Band 6 together with the SI 4 and SI 9 , as an independet variabes with EC value as dependent variable. In this correlation 6 sample points were removed from analysis and the correlation value has raised to $68.78 \%$ from $40.20 \%$ in vertical orientation.

Figure 4 shows the the distribution of residuals in the correlation analysis of the image dated 21st March, 2010.

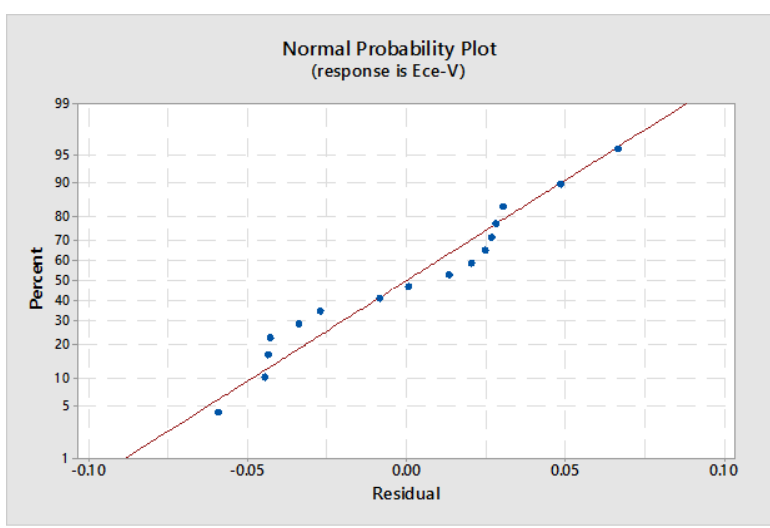

Figure 4. Multiple linear regression result of 21st March, 2010 with different combinations of bands and indices.

Regression equation obtained is given below:

Predicted Soil Salinity $=0.955-0.0406$ B $2+0.0081$ B5 $0.0370 \mathrm{~B} 6+0.0471$ SI $4-0.0454$ SI 9

\section{SOIL SALINITY MAPPING}

After all the regression methods were tested, the models with a high $\mathrm{R}^{2}$ values signifying a high correlation between satellite data and field measurements data, were selected as a best regression model in order to produce the soil salinity map of the study area. Overall the highest correlation value $(78.40 \%)$ obtained by the multiple linear regression method with all bands of the image dated 21st March, 2010 and the regression equation obtained were used for the soil mapping.

Then the density slicing method was applied in order to classify the different salinity levels. Global standard salinity ranges are taken into consideration given in the Table 5.

\begin{tabular}{|l|c|}
\hline \multicolumn{1}{|c|}{ Levels of salinity } & $\begin{array}{c}\text { Saturation extract salinity } \\
\left(\mathbf{E C}_{\mathbf{e}}, \mathbf{d S} / \mathbf{m}\right)\end{array}$ \\
\hline Non salinity & $0-2$ \\
\hline Low salinity & $2-4$ \\
\hline Medium salinity & $4-8$ \\
\hline High salinity & $8-16$ \\
\hline Extra high salinity & $>16$ \\
\hline
\end{tabular}

Table 5. Global standard salinity ranges.

The soil salinity map that indicates the salinization level according to the global standard salinity ranges (Table 5) is given in the Figure 5. As can be seen in Figure 5, only the 
small part of the Seyhan plate is being affected by salinization. The salt is mostly accumulated in the reeds region and in the other parts such as farmland; its impact can not be seen in the 2009 to 2010 periods of time.

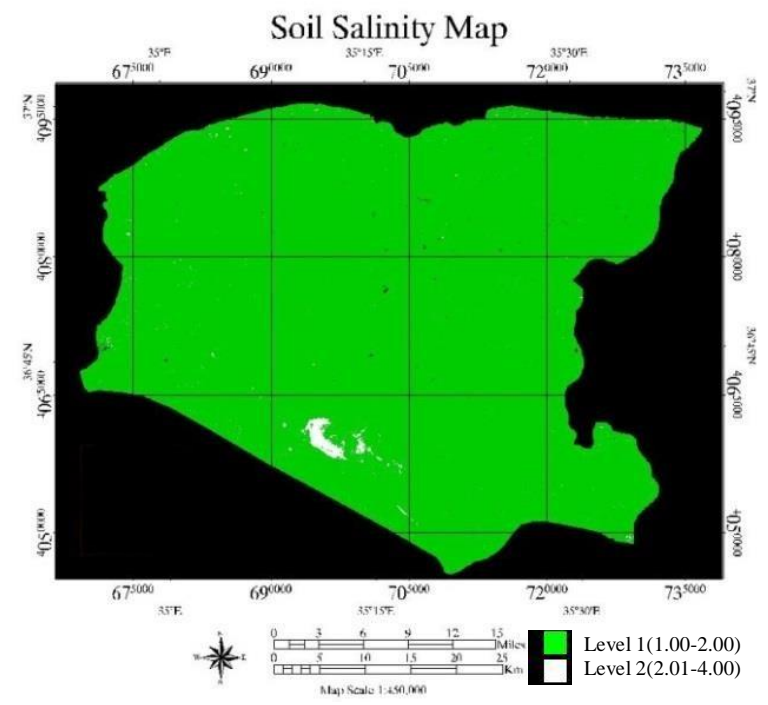

Figure 5. Soil salinity map according to global standard salinity ranges.

Alternatively, the salinity ranges used by previous research study done in this area is also considered for the soil salinity mapping. The soil salinization map produced by DSI is given in the Figure 6 (Satir, 2013).

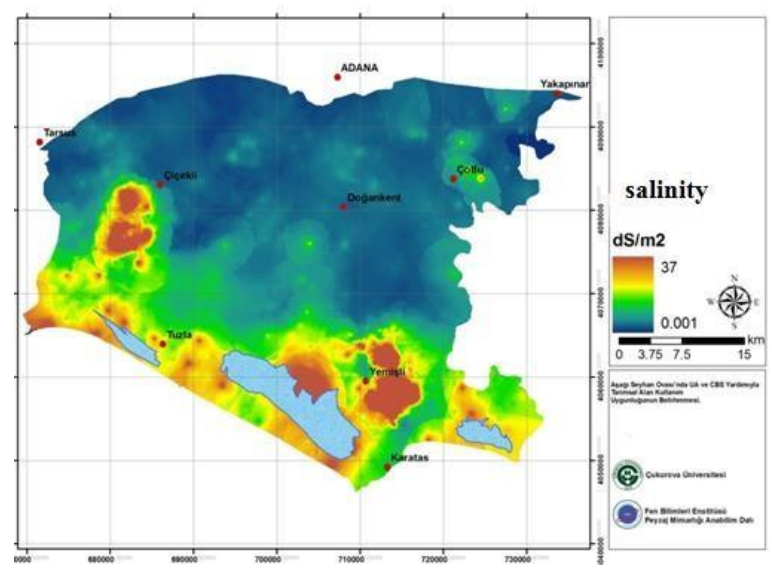

Figure 6. Soil salinity map produced by DSI.

The same process was reapplied to the highest correlated dataset (21st March, 2010) by considering the salinity ranges shown in Figure 6. Figure 7 shows the result map of density slicing process according to these ranges.

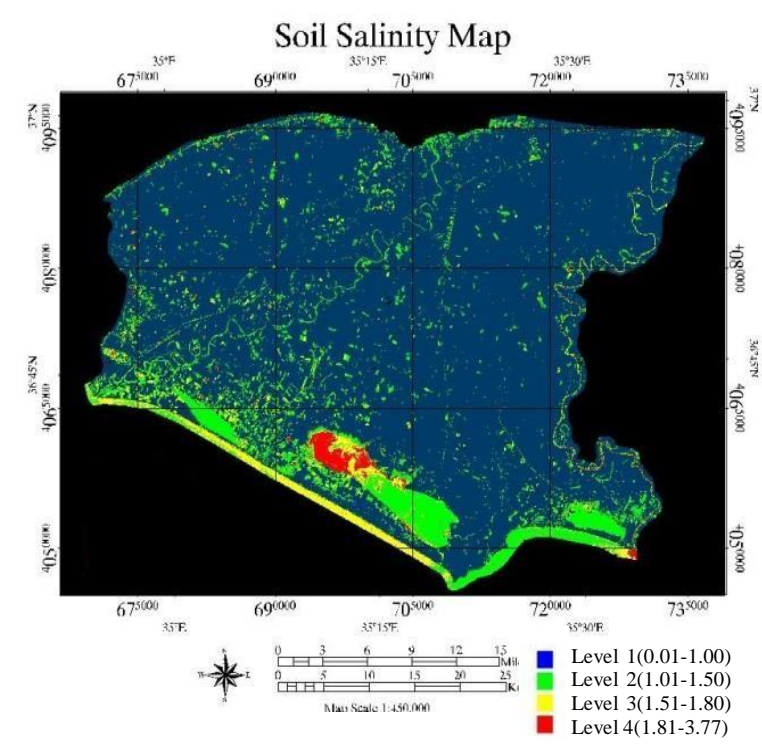

Figure 7. Soil salinity map according to DSI's ranges.

As illustrated in Figure 7, the highest saline soils in the study area again are taking place in the region covering the reeds due to the presence of high amount of salt in the lake. It can be seen that the other parts of Seyhan plate are being affected by medium to low soil salinity and the salt is mostly accumulated in the lower part of the study area.

\subsection{The analysis of salt-affected agricultural fields}

In this part of the study, intersection of the main fields with the soil salinity map was calculated to find out the percentage of the salt-affected farmlands. According to its sizes, two main fields, namely wheat and bare soil fields are taken into consideration. Map of the salt-affected wheat fields is shown in the Figure 8. As can be seen from the Figure, in general, most of the wheat fields are not much influenced by saline soil, however only the wheat fields covering lower part of the study area are affected by low to medium salinization shown by green and yellow colors.

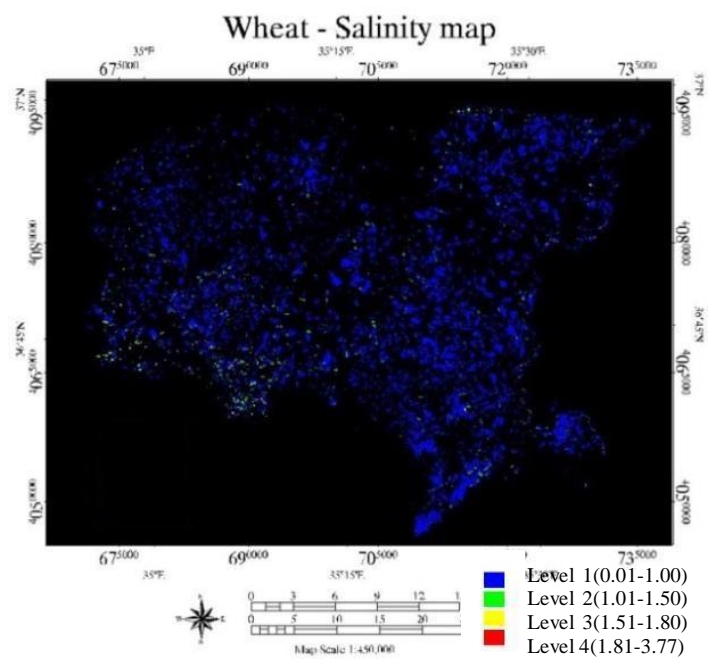

Figure 8 . The map of the salt-affected wheat fields. 
Secondly, the effect of salinization in the bare soil fields was also analyzed and the result map of the salt- affected parts is given in the Figure 9. As, can be seen, the bare soil fields, which are nearby to the reeds region, are more affected (\%35) than the other parts. In comparison with the wheat fields, it can be concluded that the bare soils are rather more influenced by salinization than the wheat fields due to having direct interaction. The areal extent of saltaffected wheat and bare soil fields are given in Table 6 .

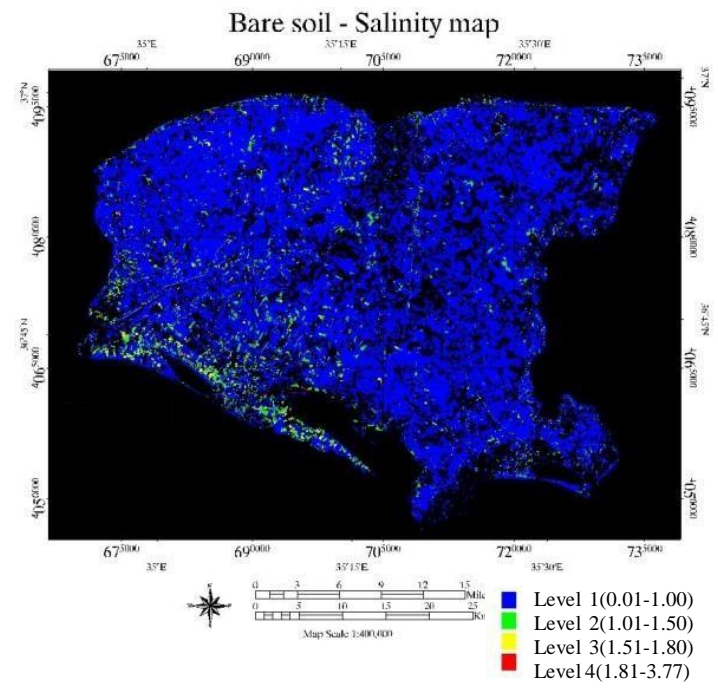

Figure 9. The map of the salt-affected bare soil fields.

\begin{tabular}{|c|c|c|c|c|}
\hline \multirow{2}{*}{$\begin{array}{l}\text { Density } \\
\text { slice ranges }\end{array}$} & \multicolumn{2}{|l|}{$\begin{array}{l}\text { Salt- affected } \\
\text { wheat fields }\end{array}$} & \multicolumn{2}{|c|}{$\begin{array}{l}\text { Salt- affected } \\
\text { bare soils fields }\end{array}$} \\
\cline { 2 - 5 } & $\mathbf{( h a )}$ & $\mathbf{( \% )}$ & $\mathbf{( h a )}$ & $\mathbf{( \% )}$ \\
\hline $\begin{array}{c}\text { Level 1 } \\
\text { (Blue) }\end{array}$ & 26242740 & 95 & 1222101000 & 56.4 \\
\hline $\begin{array}{c}\text { Level 2 } \\
\text { (Green) }\end{array}$ & 1216530 & 4 & 76045500 & 35 \\
\hline $\begin{array}{c}\text { Level 3 } \\
\text { (Yellow) }\end{array}$ & 239040 & 0.8 & 15011100 & 7 \\
\hline $\begin{array}{c}\text { Level 4 } \\
\text { (Red) }\end{array}$ & 60930 & 0.2 & 3447900 & 1.6 \\
\hline
\end{tabular}

Table 6 . The areal extent of the salt-affected wheat and salt-affected bare soils fields.

\section{RESULT AND CONCLUSIONS}

Soil salinity is a dynamic phenomenon, it can change rapidly due to the precipitation, soil moisture and evaporation; and causes major problems such as land degradation, decrease in crop production, etc. Despite the low correlations observed between ground-truth measurements and satellite image bands in many studies in the literature, the correlation analysis between the in-situ electrical conductivity measurements and multi temporal/seasonal Landsat satellite images were analyzed in the Seyhan plate, Adana by using regression techniques.
As a first approach, simple linear regression technique was applied to each individual bands and low correlation $\left(\mathrm{r}^{2}\right.$ : $30.89 \%$ to $20.02 \%$ ) was observed. As a second approach, the multiple linear regression was applied to all bands of satellite images. Among all different band combinations tested, the correlation of 18 sampled points of $21 \mathrm{st}$ March, 2010 with EC measurements, showed the highest correlation $(78.40 \%)$ due to near simultaneous acquisition of the satellite data (3 days).

After the correlation analysis, the satellite data showing the highest correlation (21st March, 2010) were chosen to map the soil salinity in the area As observed, the highest saline soils in the study area are taking place in the region covering reeds due to the presence of high amount of salt in the lake. Besides, the other parts of Seyhan plate are being affected by medium to low soil salinity and the salt is mostly accumulated in the lower part of the study area.

As a final step, the percentages of the salt-affected fields in the study area were evaluated. taking into two major fields into account, it was seen that bare soil fields are rather more influenced by salinization than the wheat fields due to having direct interaction.

Main conclusions, drawn from this study, are summarized below:

i. The compatibility between satellite data and field data; the more simultaneous satellite data and field measurements are used, the better correlation can be observed.

ii. The radiometric quality of Landsat $7 \mathrm{ETM}^{+}$; the missed lines can make it difficult to get the exact value of a pixel or visually affect the image interpretation. Although the scan line error of Landsat $7 \mathrm{ETM}^{+}$can be corrected using remote sensing software available, the original values are modified.

iii. The spatial resolution of Landsat 7 satellite (30 meters); the higher resolution makes the sampling easier in the image data.

iv. The spectral resolution of Landsat 7 satellite; Hyperspectral sensors can be a better solution, since they capture a large amount of narrow bands.

As a continuation of this study, it is planned to test the other multispectral/hyperspectral sensors data in the same area to evaluate the effectiveness of the different spectral/spatial resolutions on the salinity mapping in the future.

\section{REFERENCES}

Abbas, A. a. S. K. (2007). "Using remote sensing techniques for appraisal of irrigated soil salinity." International Congress on Modelling and Simulation of Australia and New Zealand Christchurch, New Zealand. p: 2632-8. 
Akramkhanov, A., C. Martius, S. J. Park and J. M. H. Hendrickx (2011). "Environmental factors of spatial distribution of soil salinity on flat irrigated terrain." Geoderma, 163(1-2): 55-62.

Allbed, A. and L. Kumar (2013). "Soil salinity mapping and monitoring in arid and semi-arid regions using remote sensing technology: A review." Advances in Remote Sensing 2013 (2): 373-385.

Azabdaftari, A. (2015). "Soil salinity mapping by integrating remote sensing data with ground measurements; A case study in lower seyhan plate, Adana, Turkey." Informatic Inistitute, Istanbul Technical University.

Bannari, A., A. Guedon, A. El-Harti, F. Cherkaoui and A. El-Ghmari (2008). "Characterization of Slightly and Moderately Saline and Sodic Soils in Irrigated Agricultural Land using Simulated Data of Advanced Land Imaging (EO-1) Sensor." Communications in soil science and plant analysis 39(19-20): 2795-2811.

Douaoui, A. E. K., H. Nicolas and C. Walter (2006). "Detecting salinity hazards within a semiarid context by means of combining soil and remote-sensing data." Geoderma 134(1): 217-230.

Farifteh, J., A. Farshad and R. George (2006). "Assessing salt-affected soils using remote sensing, solute modelling, and geophysics." Geoderma 130(3): 191-206.

Khan, N. M., V. V. Rastoskuev, E. V. Shalina and Y. Sato (2001). "Mapping salt-affected soils using remote sensing indicators-a simple approach with the use of GIS IDRISI." 22nd Asian Conference on Remote Sensing, 5(9).

Palialexis, A., S. Georgakarakos, I. Karakassis, K. Lika and V. Valavanis (2011). "Fish distribution predictions from different points of view: comparing associative neural networks, geostatistics and regression models." Hydrobiologia 670(1): 165-188.

Satır, O. (2013). "Aşağı Seyhan ovası'nda uzaktan algılama ve coğrafi bilgi sistemleri yardımıyla tarımsal alan kullanım uygunluğunun belirlenmesi." Graduate School of Science, Engineering and Technology, Çukurova University.

Selch, D. (2012). Comparing salinity models in Whitewater Bay using remote sensing, Florida Atlantic University. 